\title{
Common fixed point theorems for nonlinear contractions in a Menger space
}

\section{Jeong Sheok Ume*}

\section{"Correspondence:} jsume@changwon.ac.kr Department of Mathematics, Changwon National University, Changwon, 641-773, Korea

\begin{abstract}
The main purpose of this paper is to introduce a new class of Jungck-type contraction and to present some common fixed point theorems for this mapping. Several examples are given to show that our result is a proper extension of many known results.
\end{abstract}

MSC: $47 \mathrm{H} 10$

Keywords: Menger space; common fixed point theorem

\section{Introduction}

Probabilistic metric space has been introduced and studied in 1942 by Menger in USA [1], and since then the theory of probabilistic metric spaces has developed in many directions [2-6]. The idea of Menger was to use distribution functions instead of nonnegative real numbers as values of the metric. The notion of a probabilistic metric space corresponds to the situation when we do not know exactly the distance between two points, we know only probabilities of possible values of this distance. Such a probabilistic generalization of metric spaces appears to be well adapted for the investigation of physiological thresholds and physical quantities, particularly in connection with both string and $E$-infinity which were introduced and studied by a well-known scientific hero El Naschie [7-9].

It is observed by many authors that the contraction condition in a metric space may be exactly translated into a probabilistic metric space endowed with min norms. Sehgal and Bharucha-Reid [10] obtained a generalization of the Banach contraction principle on a complete Menger space, which is a milestone in developing fixed point theorems in a Menger space.

Jungck's fixed point theorem [11] has many applications in nonlinear analysis. This theorem is extended by several authors; see [12-16] and the references therein.

In this paper, we introduce a new class of Jungck-type contraction and present some common fixed point theorems for this mapping. Several examples are given to show that our result is a proper extension of many known results.

\section{Preliminaries}

Throughout this paper we denote by $N$ the set of all positive integers, by $Q$ the set of all rational numbers, by $Z^{+}$the set of all nonnegative integers, by $R$ the set of all real numbers and by $R^{+}$the set of all nonnegative real numbers. We shall recall some definitions and lemmas related to a Menger space.

( 2013 Ume; licensee Springer. This is an Open Access article distributed under the terms of the Creative Commons Attribution License (http://creativecommons.org/licenses/by/2.0), which permits unrestricted use, distribution, and reproduction in any medium, provided the original work is properly cited. 
Definition 2.1 A mapping $F: R \rightarrow R^{+}$is called a distribution if it is nondecreasing left continuous with $\inf \{F(t): t \in R\}=0$ and $\sup \{F(t): t \in R\}=1$. We shall denote by $L$ the set of all distribution functions. The specific distribution function $H: R \rightarrow R^{+}$is defined by

$$
H(t)= \begin{cases}0, & t \leq 0, \\ 1, & t>0 .\end{cases}
$$

Definition 2.2 ([13]) Probabilistic metric space (PM-space) is an ordered pair $(X, F)$, where $X$ is an abstract set of elements and $F: X \times X \rightarrow L$ is defined by $(p, q) \rightarrow F_{p, q}$, where $\left\{F_{p, q}: p, q \in X\right\} \subseteq L$, where the functions $F_{p, q}$ satisfy the following:

(a) $F_{p, q}(x)=1$ for all $x>0$ if and only if $p=q$;

(b) $F_{p, q}(0)=0$;

(c) $F_{p, q}=F_{q, p}$;

(d) $F_{p, q}(x)=1$ and $F_{q, r}(y)=1$, then $F_{p, r}(x+y)=1$.

Definition 2.3 A mapping $t:[0,1] \times[0,1] \rightarrow[0,1]$ is called a $t$-norm if

(e) $t(0,0)=0$ and $t(a, 1)=a$ for all $a \in[0,1]$;

(f) $t(a, b)=t(b, a)$ for all $a, b \in[0,1]$;

(g) $t(a, b) \leq t(c, d)$ for all $a, b, c, d \in[0,1]$ with $a \leq c$ and $b \leq d$;

(h) $t(t(a, b), c)=t(a, t(b, c))$ for all $a, b, c \in[0,1]$.

Definition 2.4 A Menger space is a triplet $(X, F, t)$, where $(X, F)$ is a PM-space and $t$ is a $t$-norm such that for all $p, q, r \in X$ and all $x, y \geq 0$,

$$
F_{p, r}(x+y) \geq t\left(F_{p, q}(x), F_{q, r}(y)\right) .
$$

Definition 2.5 ([13]) Let $(X, F, t)$ be a Menger space and $f: X \rightarrow X$.

(1) A sequence $\left\{p_{n}\right\}$ in $X$ is said to converge to a point $p$ in $X$ (written as $p_{n} \rightarrow p$ ) if for every $\varepsilon>0$ and $\lambda>0$, there exists a positive integer $M(\varepsilon, \lambda)$ such that $F_{p_{n}, p}(\varepsilon)>1-\lambda$ for all $n \geq M(\varepsilon, \lambda)$.

(2) A sequence $\left\{p_{n}\right\}$ in $X$ is said to be Cauchy if for each $\varepsilon>0$ and $\lambda>0$, there is a positive integer $M(\varepsilon, \lambda)$ such that $F_{p_{n}, p_{m}}(\varepsilon) \geq 1-\lambda$ for all $n, m \in N$ with $n, m \geq M(\varepsilon, \lambda)$.

(3) A Menger space $(X, F, t)$ is said to be complete if every Cauchy sequence in $X$ converges to a point of it.

(4) $f$ is said to be continuous at a point $p$ in $X$ if for every sequence $\left\{p_{n}\right\}$ in $X$, which converges to $p$, the sequence $\left\{f\left(p_{n}\right)\right\}$ in $X$ converges to $f(p)$.

(5) $f$ is said to be continuous on $X$ if $f$ is continuous at every point in $X$.

Definition 2.6 ([4]) A $t$-norm $t$ is said to be of $H$-type if a family of functions $\left\{t^{n}(a)\right\}_{n=1}^{\infty}$ is equicontinuous at $a=1$, that is, for any $\varepsilon \in(0,1)$, there exists $\delta \in(0,1)$ such that $a>1-\delta$ and $n \geq 1$ imply $t^{n}(a)>1-\varepsilon$. The $t$-norm $t=\min$ is a trivial example of a $t$-norm of $H$-type, but there are $t$-norms of $H$-type with $t$-norm $\neq \min$ (see, e.g., Hadzic [5]).

From Definition 2.1-Definition 2.5, we can prove easily the following lemmas.

Lemma 2.7 ([10]) If $(X, d)$ is a metric, then the metric induces a mapping $X \times X \rightarrow L$, defined by $F_{p, q}(x)=H(x-d(p, q)), p, q \in X$ and $x \in R$. Further, if the $t$-norm $t:[0,1] \times$ 
$[0,1] \rightarrow[0,1]$ is defined by $t(a, b)=\min \{a, b\}$ for all $a, b \in[0,1]$, then $(X, F, t)$ is a Menger space. It is complete if $(X, d)$ is complete.

Lemma 2.8 In a Menger space $(X, F, t)$, if $t(x, x) \geq x$ for all $x \in[0,1]$, then $t(a, b)=\min \{a, b\}$ for all $a, b \in[0,1]$.

\section{Jungck-type fixed point theorems}

In 1976, Jungck proved the following theorem.

Theorem A (Jungck [11], 1976) Let $f$ be a continuous mapping of a complete metric space $(X, d)$ into itself and let $g: X \rightarrow X$ be a map that satisfies the following conditions:
(a) $g(X) \subseteq f(X)$;
(b) g commutes withf;
(c) $\quad d(g(x), g(y)) \leq k d(f(x), f(y))$

for all $x, y \in X$ and for some $0<k<1$. Then $f$ and $g$ have a unique common fixed point.

Definition 3.1 Let $(X, F, t)$ be a Menger space with $t(x, x) \geq x$ for all $x \in[0,1]$ and let $f, g$ : $X \rightarrow X$ be two self-mappings of $X$. We will say that $f$ and $g$ are Jungck-type generalized contraction if

$$
F_{g(p), g(q)}(\varphi(x)) \geq F_{f(p), f(q)}(x)
$$

for all $p, q \in X$ and $x>0$, where $\varphi:[0, \infty) \rightarrow[0, \infty)$ is a mapping such that $\varphi(x)<x$ for all $x>0$, and for all $p, q \in X$ and $x \in R, F_{p, q}(x)$ is the same as in Definition 2.2.

\section{Remark 3.2}

(1) It is clear that $(* 1)$ implies ( $* 2)$ if $F_{p, q}(x)=H(x-d(p, q))$ for all $p, q \in X, x \in R$, and $\varphi(x)=k x$ for all $x \in R^{+}$, where $0<k<1$.

(2) In Example 3.10, we shall show that the condition $(* 2)$ is satisfied, but the condition $(* 1)$ is not satisfied.

Definition 3.3 Let $\varphi:[0, \infty) \rightarrow[0, \infty)$ be a mapping such that $\varphi(x)<x$ for all $x>0$. We say that $\varphi$ is the $U$-generalized contraction if

$$
\varphi\left((x-\varphi(x))\left(\frac{\varphi(x)}{x}\right)^{r}\right) \leq(x-\varphi(x))\left(\frac{\varphi(x)}{x}\right)^{r+1}
$$

for all $x>0$ and $r \in Z^{+}$.

Lemma 3.4 Let $k \in(0,1)$ be as in (c) of Theorem A and let $\varphi:[0, \infty) \rightarrow[0, \infty)$ be defined by

$$
\varphi(x)= \begin{cases}\left(\frac{1+k}{2}\right) x+\left(\frac{1-k}{2}\right) x^{2}, & 0 \leq x \leq k, \\ \left(\frac{1}{2}+k-\frac{k^{2}}{2}\right) x, & k<x .\end{cases}
$$

Then $\varphi$ is an U-generalized contraction. 
Proof It follows from hypotheses that for all $x>0$,

$$
\varphi(x)<x
$$

Now we shall show that condition $(* 3)$ is satisfied. Since

$$
(x-\varphi(x))\left(\frac{\varphi(x)}{x}\right)^{r} \leq k \quad \text { for all } x \in(0, k] \text { and } r \in Z^{+},
$$

there are three cases which need to be considered.

Case 1. Let $x \in(0, k]$ and $r \in Z^{+}$. Then, since

$$
\left(1-\frac{\varphi(x)}{x}\right)\left(\frac{\varphi(x)}{x}\right)^{r} \leq 1
$$

$(* 3)$ is satisfied.

Case 2. Let $x \in(k, \infty)$ and $r \in Z^{+}$with $(x-\varphi(x))\left(\frac{\varphi(x)}{x}\right)^{r} \leq k$. Then, since

$$
\left(\frac{1+k}{2}\right)+\left(\frac{1-k}{2}\right)(x-\varphi(x))\left(\frac{\varphi(x)}{x}\right)^{r} \leq \frac{1}{2}+k-\frac{k^{2}}{2},
$$

$(* 3)$ is satisfied.

Case 3. Let $x \in(k, \infty)$ and $r \in Z^{+}$with $k<(x-\varphi(x))\left(\frac{\varphi(x)}{x}\right)^{r}$. Then, since

$$
\frac{1}{2}+k-\frac{k^{2}}{2}=\frac{\varphi(x)}{x}
$$

$(* 3)$ is satisfied. From $(* 4)$, Case 1 , Case 2 and Case $3, \varphi$ is $U$-generalized contraction.

The following example shows that $f$ and $g$ do not have a common fixed point even though $f, g$ and $\varphi$ satisfy $(* 2)$ and $(* 3)$.

Example 3.5 Let $k \in(0,1)$ and $\varphi:[0, \infty) \rightarrow[0, \infty)$ be as in Lemma 3.4. Let $f, g: R \rightarrow R$ be defined by $f(x)=x+1$ and $g(x)=\frac{k}{2} x$. Define $F_{p, q}: R \rightarrow R^{+}$by

$$
F_{p, q}(x)=H(x-|p-q|) \quad \text { for all } p, q \in R \text { and } x \in R,
$$

where $F_{p, g}$ and $H$ are the same as in Definition 2.1 and Definition 2.2. Let $t:[0,1] \times[0,1] \rightarrow$ $[0,1]$ be defined by $t(a, b)=\min \{a, b\}$ for all $a, b \in[0,1]$. Then, by Lemma 3.4 and simple calculations, $(* 2)$ and $(* 3)$ are satisfied. But $f$ and $g$ do not have a common fixed point.

Remark 3.6 It follows from Example 3.5 that $f$ and $g$ must satisfy $(* 2)$ and $(* 3)$, and other conditions additionally in order to have a common fixed point of $f$ and $g$.

The following is Jungck-type common fixed point theorem which is a generalization of Jungck's common fixed point theorem [11]. 
Theorem 3.7 Let $(X, F, t)$ be a complete Menger space with continuous t-norm and $t(x, x) \geq x$ for all $x \in[0,1]$, let $f$ be a continuous self-mapping on $X$ and let $\varphi: R^{+} \rightarrow R^{+}$ be a mapping that satisfies the following conditions:

(i) $g(X) \subseteq f(X)$;

(ii) $g$ commutes with $f$;

(iii) $f, g$ and $\varphi$ satisfy $(* 2)$ and $(* 3)$;

(iv) $\varphi$ is a strictly increasing and bijective;

(v) $\lim _{n \rightarrow \infty} \varphi^{-n}(x)=\infty$ for each $x>0$, where $\varphi^{-n}$ is n-times repeated composition of $\varphi^{-1}$ with itself.

Then $f$ and $g$ have a unique common fixed point.

Proof

It is easy to see that the self-mapping $g$ on $X$ in Theorem 3.7 is continuous on $X$.

Let $x_{0} \in X$. By (i), there exists a sequence $\left\{x_{n}\right\}_{n=0}^{\infty}$ in $X$ such that

$$
f\left(x_{n}\right)=g\left(x_{n-1}\right) \quad \text { for all } n \in N \text {. }
$$

From (iii) and (3.2), we have

$$
F_{g\left(x_{n-1}\right), g\left(x_{n}\right)}(\varphi(x)) \geq F_{f\left(x_{n-1}\right), f\left(x_{n}\right)}(x) \quad \text { for all } n \in N \text { and } x>0 .
$$

By virtue of (iv), (3.2) and (3.3), we obtain

$$
F_{f\left(x_{n}\right), f\left(x_{n+1}\right)}(x)=F_{g\left(x_{n-1}\right), g\left(x_{n}\right)}(x) \geq F_{f\left(x_{n-1}\right), f\left(x_{n}\right)}\left(\varphi^{-1}(x)\right)
$$

for all $n \in N$ and $x>0$. In view of (3.4), we have

$$
F_{f\left(x_{n}\right), f\left(x_{n-1}\right)}(x) \geq F_{f\left(x_{0}\right), f\left(x_{1}\right)}\left(\varphi^{-n}(x)\right)
$$

for all $n \in N$ and $x>0$. By repeated application of (3.5), we have

$$
F_{f\left(x_{n+j}\right) f\left(x_{n+1+j}\right)}(x) \geq F_{f\left(x_{n}\right), f\left(x_{n+1}\right)}\left(\varphi^{-j}(x)\right)
$$

for all $n, j \in N$ and $x>0$. From (iii), we have

$$
0<\frac{\varphi(x)}{x}<1 \quad \text { for all } x>0 .
$$

On account of (3.7), we obtain that

$$
\sum_{k=0}^{\infty}\left[\frac{\varphi(x)}{x}\right]^{k}=\frac{1}{1-\left(\frac{\varphi(x)}{x}\right)} \quad \text { for all } x>0
$$


In terms of (3.8), we get that

$$
x=(x-\varphi(x)) \sum_{k=0}^{\infty}\left(\frac{\varphi(x)}{x}\right)^{k} \text { for all } x>0
$$

Now we shall show that $\left\{f\left(x_{n}\right)\right\}$ is a Cauchy sequence.

Let $n, m \in N$ be such that $n<m$.

From (iii), (iv), (3.5)-(3.10) and Definition 2.4, we deduce that

$$
\begin{aligned}
F_{f\left(x_{n}\right), f\left(x_{m}\right)}(x) & \\
= & F_{f\left(x_{n}\right), f\left(x_{m}\right)}\left((x-\varphi(x)) \sum_{k=0}^{\infty}\left(\frac{\varphi(x)}{x}\right)^{k}\right) \\
\geq & F_{f\left(x_{n}\right), f\left(x_{m}\right)}\left((x-\varphi(x)) \sum_{k=0}^{m-n-1}\left(\frac{\varphi(x)}{x}\right)^{k}\right) \\
\geq & \min \left\{F_{f\left(x_{n}\right), f\left(x_{n+1}\right)}((x-\varphi(x))),\right. \\
& F_{f\left(x_{n+1}\right), f\left(x_{n+2}\right)}\left((x-\varphi(x))\left(\frac{\varphi(x)}{x}\right)\right), \ldots, \\
& \left.F_{f\left(x_{m-1}\right), f\left(x_{m}\right)}\left((x-\varphi(x))\left(\frac{\varphi(x)}{x}\right)^{m-n-1}\right)\right\} \\
\geq & \min \left\{F_{f\left(x_{n}\right), f\left(x_{n+1}\right)}((x-\varphi(x))),\right. \\
& F_{f\left(x_{n}\right), f\left(x_{n+1}\right)}\left(\varphi^{-1}\left((x-\varphi(x))\left(\frac{\varphi(x)}{x}\right)\right)\right), \ldots, \\
\geq & F_{f\left(x_{n}\right), f\left(x_{n+1}\right)}\left(\varphi_{f\left(x_{0}\right), f\left(x_{1}\right)}\left(\varphi^{-n}(x-\varphi(x))\right)\right. \\
& F_{f\left(x_{n}\right), f\left(x_{n+1}\right)}((x-\varphi(x))) \\
&
\end{aligned}
$$

for all $x>0$ and $n, m \in N$ with $n<m$. In terms of (iii), (v) and Definition 2.2, we have

$$
\lim _{n \rightarrow \infty} F_{f\left(x_{0}\right), f\left(x_{1}\right)}\left(\varphi^{-n}(x-\varphi(x))\right)=1 \quad \text { for all } x>0 .
$$

By (3.11), (3.12) and Definition 2.2, $\left\{f\left(x_{n}\right)\right\}$ is a Cauchy sequence in $X$. Since $X$ is complete and $\left\{f\left(x_{n}\right)\right\}$ is a Cauchy sequence in $X$, there exists $z \in X$ such that

$$
\lim _{n \rightarrow \infty} f\left(x_{n}\right)=z
$$

On account of (3.2) and (3.13), we have

$$
\lim _{n \rightarrow \infty} g\left(x_{n}\right)=z
$$


By (ii), (3.1), (3.13), (3.14) and hypotheses,

$$
\begin{aligned}
& f\left(g\left(x_{n}\right)\right)=g\left(f\left(x_{n}\right)\right) \quad \text { for all } n \in N, \\
& \lim _{n \rightarrow \infty} f\left(g\left(x_{n}\right)\right)=f(z)
\end{aligned}
$$

and

$$
\lim _{n \rightarrow \infty} g\left(f\left(x_{n}\right)\right)=g(z)
$$

From (3.15), we get that

$$
f(z)=g(z) .
$$

In view of (ii), (3.16) and $(* 2)$, we have

$$
\begin{aligned}
F_{g(z), g(g(z))}(\varphi(x)) & \geq F_{f(z), f(g(z))}(x) \geq F_{g(z), g(f(z))}(x) \\
& \geq F_{g(z), g(g(z))}(x)
\end{aligned}
$$

for all $x>0$.

By (iv) and (3.17),

$$
F_{g(z), g(g(z))}(x) \geq F_{g(z), g(g(z))}\left(\varphi^{-n}(x)\right)
$$

for all $n \in N$ and $x>0$. Due to (v), (3.18), Definition 2.1 and Definition 2.2, we get that

$$
g(z)=g(g(z))
$$

From (ii), (3.16) and (3.19), we have

$$
g(z)=g(g(z))=g(f(z))=f(g(z)) .
$$

By (3.20), $g(z)$ is a common fixed point of $f$ and $g$. To prove the uniqueness of a common fixed point of $f$ and $g$, let $u$ and $w$ be common fixed points of $f$ and $g$. Then $f(u)=g(u)=u$ and $f(w)=g(w)=w$. Putting $p=u$ and $q=w$ in $(* 2)$, we get

$$
F_{g(u), g(w)}(\varphi(x))=F_{u, w}(\varphi(x)) \geq F_{f(u), f(w)}(x)=F_{u, w}(x)
$$

for all $x>0$, which gives $u=w$. Thus $g(z)$ is a unique common fixed point of $f$ and $g$.

Now we give an example to support Theorem 3.7.

Example 3.8 Let $X=R$ be the set of reals with the usual metric and let $f, g: X \rightarrow X$ and $\varphi: R^{+} \rightarrow R^{+}$be mappings defined as follows:

$$
f(x)=3 x, \quad g(x)=2 x \quad \text { and } \quad \varphi(x)= \begin{cases}\frac{2}{3} x+\frac{1}{3} x^{2}, & 0 \leq x \leq \frac{2}{3} \\ \frac{8}{9} x, & \frac{2}{3}<x\end{cases}
$$


Let the mappings $F_{p, q}, H$ and $t$ be as in Example 3.5. Then from Lemma 2.7, $(X, F, t)$ is a complete Menger space. By the same method as in Lemma 3.4 and simple calculations, the conditions of Theorem 3.7 are satisfied. Thus $f$ and $g$ have a unique common fixed point 0 .

From Theorem 3.7, we have the following corollary.

Corollary 3.9 Let $(X, F, t)$ be a complete Menger space with continuous $t$-norm and $t(x, x) \geq x$ for all $x \in[0,1]$. Let $f, g:$ ' $X \rightarrow X$ be maps that satisfy the following conditions:

(a) $g(X) \subseteq f(X)$;

(b) $f$ is continuous;

(c) $g$ commutes with $f$;

(d) $F_{g(p), g(q)}(k x) \geq F_{f(p), f(q)}(x)$ for all $p, q \in X, x>0$ and for some $0<k<1$. Then $f$ and $g$ have a unique common fixed point.

Proof Let $\varphi: R^{+} \rightarrow R^{+}$be defined by

$$
\varphi(x)=k x, \quad 0<k<1 .
$$

From (b) and (d), we deduce that $g$ is continuous. Thus, by (3.23), the same method as in Lemma 3.4 and simple calculations, the conditions of Theorem 3.7 are satisfied. Therefore $f$ and $g$ have a unique common fixed point.

In the next example, we shall show that all the conditions of Theorem 3.7 are satisfied, but condition (d) in Corollary 3.9 and condition $(* 1)$ in Theorem A are not satisfied.

Example 3.10 Let $k \in(0,1)$ be as in (c) of Theorem A and let $X=R$ be the set of reals with usual metric. Suppose that $f, g: X \rightarrow X$ and $\varphi: R^{+} \rightarrow R^{+}$are mappings defined as follows:

$$
f(x)=k x, \quad g(x)=\left(\frac{k+k^{2}}{2}\right) x
$$

and

$$
\varphi(x)= \begin{cases}\left(\frac{1+k}{2}\right) x+\left(\frac{1-k}{2}\right) x^{2}, & 0 \leq x \leq k, \\ \left(\frac{1}{2}+k-\frac{k^{2}}{2}\right) x, & k<x .\end{cases}
$$

Let the mappings $F_{p, q}, H$ and $t$ be the same as in Example 3.5. Then, from Lemma 2.7 and Lemma $3.4,(X, F, t)$ is a complete Menger space and $\varphi$ satisfies $(* 3)$. Since

$$
|g(p)-g(g)| \leq \varphi(|f(p)-f(g)|)
$$

for all $p, q \in X$, we deduce that

$$
F_{g(p), g(q)}(\varphi(x)) \geq F_{f(p), f(q)}(x)
$$

for all $p, q \in X$ and $x>0$, which implies ( $* 2$ ). By simple calculations, conditions (i), (ii), (iv) and (v) of Theorem 3.7 are satisfied. Thus all the conditions of Theorem 3.7 are satisfied. 
Hence $f$ and $g$ have a unique common fixed point 0 . By hypotheses, there exist $p_{1}=-k \in R$, $q_{1}=0 \in R$ and $x_{1}=\frac{3}{4} k^{2}+\frac{1}{4} k>0$ such that

$$
F_{g\left(p_{1}\right), g\left(q_{1}\right)}\left(k x_{1}\right)<F_{f\left(p_{1}\right), f\left(q_{1}\right)}\left(x_{1}\right)
$$

which implies that condition (d) of Corollary 3.9 is not satisfied. By hypotheses, there exist $p_{2}=-k^{2} \in R$ and $q_{2}=0 \in R$ such that

$$
\left|g\left(p_{2}\right)-g\left(q_{2}\right)\right|>k\left|f\left(p_{2}\right)-f\left(q_{2}\right)\right|
$$

which implies that condition $(* 1)$ in Theorem A is not satisfied. Therefore Theorem 3.7 is a proper extension of Theorem A and Corollary 3.9.

\section{A natural question arises from Example 3.5.}

Question Would Theorem 3.7 remain true if (i)-(v) in Theorem 3.7 were substituted by some suitable conditions?

\section{Competing interests}

The author declares that he has no competing interests.

\section{Authors' contributions}

The author completed the paper himself. The author read and approved the final manuscript.

\section{Acknowledgements}

The author would like to thank referees for careful reading and useful comments. This research was supported by Basic

Science Research Program through the National Research Foundation of Korea (NRF) funded by the Ministry of

Education, Science and Technology (2012R1A1A2002165).

Received: 6 September 2012 Accepted: 4 June 2013 Published: 26 June 2013

\section{References}

1. Menger, K: Statistical metrics. Proc. Natl. Acad. Sci. USA 28, 535-537 (1942)

2. Chang, SS, Cho, YJ, Kang, SM: Nonlinear Operator Theory in Probabilistic Metric Space. Nova Science Publishers, New York (2001)

3. Cho, YJ, Ha, KS, Chang, SS: Common fixed point theorems for compatible mappings of type (A) in non-Archimedean Menger PM-spaces. Math. Jpn. 46, 169-179 (1997)

4. Hadzić, O: A fixed point theorem in Menger spaces. Publ. Inst. Math. (Belgr.) 26, 107-112 (1979)

5. Hadzić, O: Fixed point theorems for multivalued mappings in probabilistic metric spaces. Fuzzy Sets Syst. 88, 219-226 (1997)

6. Schweizer, B, Sklar, A: Probabilistic Metric Spaces. North-Holand Series in Probability and Applied Mathematics. North-Holand, New York (1983)

7. El Naschie, MS: On the uncertainty of Cantorian geometry and two-slit experiment. Chaos Solitons Fractals 9 , 517-529 (1998)

8. El Naschie, MS: A review of E-infinity theory and the mass spectrum of high energy particle physics. Chaos Solitons Fractals 19, 209-236 (2004)

9. El Naschie, MS: The idealized quantum two-slit Gedanken experiment revisited-criticism and reinterpretation. Chaos Solitons Fractals 27, 9-13 (2006)

10. Sehgal, VM, Bharucha-Reid, AT: Fixed points of contraction mappings on probabilistic metric spaces. Math. Syst. Theory 6, 97-102 (1972)

11. Jungck, G: Commuting maps and fixed points. Am. Math. Mon. 83, 261-263 (1976)

12. Jungck, G: Common fixed points for commuting and compatible maps on compacta. Proc. Am. Math. Soc. 103, 977-983 (1988)

13. Mishra, SN: Common fixed points of compatible mappings in PM-spaces. Math. Jpn. 36, 283-289 (1991)

14. Razani, A, Fouladgar, K: Extension of contractive maps in the Menger probabilistic quasi-metric space. Chaos Solitons Fractals 34, 1724-1731 (2007)

15. Rezaiyan, R, Cho, YJ, Saadati, R: A common fixed point theorems in Menger probabilistic quasi-metric spaces. Chaos Solitons Fractals 37, 1153-1157 (2008)

16. Saadati, R, Vaezpour, SM, Ćirić, LB: Generalized distance and some common fixed point theorems. J. Comput. Anal. Appl. 12, 157-162 (2010) 
doi:10.1186/1687-1812-2013-166

Cite this article as: Ume: Common fixed point theorems for nonlinear contractions in a Menger space. Fixed Point Theory and Applications 2013 2013:166

Submit your manuscript to a SpringerOpen ${ }^{\circ}$ journal and benefit from:

- Convenient online submission

- Rigorous peer review

- Immediate publication on acceptance

- Open access: articles freely available online

- High visibility within the field

- Retaining the copyright to your article

Submit your next manuscript at $\gg$ springeropen.com 